\title{
A practical method for an efficient and optimal production of selenomethionine- labeled recombinant protein complexes in the insect cells
}

\author{
Sabine Wenzel, Tsuyoshi Imasaki, and Yuichiro Takagi \\ Department of Biochemistry and Molecular Biology, Indiana University School of \\ Medicine, 635 Barnhill Drive, Indianapolis, Indiana 46202, USA
}

\begin{abstract}
:
Single or multi-wavelength anomalous diffraction (SAD or MAD) phasing using Selenomethionine (SeMet) incorporated protein crystals has become almost synonymous in a modern X-ray crystallography. Anomalous signals from SeMets can be used for phasing as well as markers for model building. SeMet incorporated recombinant proteins can be obtained in large quantities when expressed in E. coli. In contrast, a production of SeMet labeled recombinant proteins expressed in insect cells is not as robust due to toxicity of SeMet to the insect cells. Previously reported protocols for SeMet-incorporation in insect cells have proven to be rather strenuous, suited more for secreted proteins, and have, for the most part, not addressed SeMet toxicity issue, resulting typically in low recovery of the labeled proteins. We found that SeMet toxicity can be circumvented fully infecting insect cells with baculocvirus. Quantitatively controlling infection using our Titer Estimation of Quality Control (TEQC) method allows for incorporation of substantial amounts of SeMet, resulting in an efficient and optimal production of labeled recombinant protein complexes. With the method described here, we were able to reach incorporation levels of about $75 \%$ and protein yield of $60-90 \%$ compared to native protein expression consistently.
\end{abstract}

